\title{
Erratum to "An Inventory Model for Deteriorating Items with Generalised Exponential Decreasing Demand, Constant Holding Cost and Time-Varying Deterioration Rate" [American Journal of Operations Research 8 (2018) 1-16]
}

\author{
Isiyaku Aliyu' ${ }^{1}$, Babangida Sani $^{2}$ \\ ${ }^{1}$ Department of Mathematics and Statistics, Kaduna Polytechnic, Kaduna, Nigeria \\ ${ }^{2}$ Department of Mathematics, Ahmadu Bello University, Zaria, Nigeria \\ Email: isiyakualiyu30@gmail.com
}

How to cite this paper: Aliyu, I. and Sani, B. (2021) Erratum to "An Inventory Model for Deteriorating Items with Generalised Exponential Decreasing Demand, Constant Holding Cost and Time-Varying Deterioration Rate" [American Journal of Operations Research 8 (2018) 1-16]. American Journal of Operations Research, 11, 100-109. https://doi.org/10.4236/ajor.2021.112006

Received: December 28, 2020

Accepted: March 15, 2021

Published: March 18, 2021

Copyright $\odot 2021$ by author(s) and Scientific Research Publishing Inc. This work is licensed under the Creative Commons Attribution International License (CC BY 4.0).

http://creativecommons.org/licenses/by/4.0/ (c) (i) Open Access
The original online version of this article (Isiyaku Aliyu, Babangida Sani (2018) [18] An Inventory Model for Deteriorating Items with Generalised Exponential Decreasing Demand, Constant Holding Cost and Time-Varying Deterioration Rate. AJOR, 8, 1-16. doi: 10.4236/ajor.2018.81001.) was published as some results data reported mistakenly. The author wishes to correct the errors to:

In solving Equation (1) of the paper, which described the state of the inventory level at any time, $t$, in the inventory cycle, a mistake was made. We now solve the equation again so as to correct the mistake.

The differential equation is given as

$$
\frac{\mathrm{d} I(t)}{\mathrm{d} t}+(a+b t) I(t)=-D(t), 0 \leq t \leq T
$$

where $D(t)=K \mathrm{e}^{h-\beta t}$

To determine the correct solution of the equation, we proceed as follows: $\frac{\mathrm{d} I(t)}{\mathrm{d} t}+I(t)(a+b t)=-K \mathrm{e}^{h-\beta t}$. This is a linear differential equation where the Integrating factor is $\mathrm{e}^{a t+\frac{1}{2} b t^{2}}$ so that the solution is 


$$
I(t) \mathrm{e}^{a t+\frac{1}{2} b t^{2}}=-K \int \mathrm{e}^{h-\beta t+a t+\frac{1}{2} b t^{2}} \mathrm{~d} t
$$

In trying to determine the integral on the right hand side of Equation (2), it is noted that the exponent is not linear so the exponential cannot be integrated directly. One needs to use the series solution of the exponential and then integrate term-by-term (see Wataru, 2014 [19]).

The Integral then becomes

$$
\begin{aligned}
= & -K \int\left[1+\left(h-\beta t+a t+\frac{1}{2} b t^{2}\right)+\frac{\left(h-\beta t+a t+\frac{1}{2} b t^{2}\right)^{2}}{2 !}+\cdots\right] \mathrm{d} t \\
= & -K \int\left[1+h-\beta t+a t+\frac{1}{2} b t^{2}+\frac{h^{2}}{2}-h \beta t+h a t+\frac{b h t^{2}}{2}+\frac{\beta^{2} t^{2}}{2}\right. \\
& \left.-\beta a t^{2}-\frac{\beta b t^{3}}{2}+\frac{a b t^{3}}{2}+\frac{a^{2} t^{2}}{2}+\frac{b^{2} t^{4}}{8}+\cdots\right] \mathrm{d} t \\
= & -K\left[t+h t-\frac{\beta t^{2}}{2}+\frac{a t^{2}}{2}+\frac{b t^{3}}{6}+\frac{h^{2} t}{2}-\frac{h \beta t^{2}}{2}+\frac{h a t^{2}}{2}+\frac{b h t^{3}}{6}\right. \\
& \left.+\frac{\beta^{2} t^{3}}{6}-\frac{\beta a t^{3}}{3}-\frac{\beta b t^{4}}{8}+\frac{a b t^{4}}{8}+\frac{a^{2} t^{3}}{6}+\frac{b^{2} t^{5}}{40}+\cdots\right]+Q
\end{aligned}
$$

where $Q$ is a constant.

Applying the initial boundary condition i.e at $t=0, I(t)=I_{0}$

$$
I_{0} \mathrm{e}^{0}=-K[0]+Q \text { i.e } Q=I_{0}
$$

Substituting Equation (5) into Equation (4), we get

$$
\begin{aligned}
I(t) \mathrm{e}^{a t+\frac{1}{2} b t^{2}}= & -K\left[t+h t-\frac{\beta t^{2}}{2}+\frac{a t^{2}}{2}+\frac{b t^{3}}{6}+\frac{h^{2} t}{2}-\frac{h \beta t^{2}}{2}+\frac{h a t^{2}}{2}+\frac{b h t^{3}}{6}\right. \\
& \left.+\frac{\beta^{2} t^{3}}{6}-\frac{\beta a t^{3}}{3}-\frac{\beta b t^{4}}{8}+\frac{a b t^{4}}{8}+\frac{a^{2} t^{3}}{6}+\frac{b^{2} t^{5}}{40}+\cdots\right]+I_{0}
\end{aligned}
$$

Applying the boundary condition at $t=T, I(t)=0$ into Equation (6) gives

$$
\begin{aligned}
0= & -K\left[T+h T-\frac{\beta T^{2}}{2}+\frac{a T^{2}}{2}+\frac{b T^{3}}{6}+\frac{h^{2} T}{2}-\frac{h \beta T^{2}}{2}+\frac{h a T^{2}}{2}+\frac{b h T^{3}}{6}\right. \\
& \left.+\frac{\beta^{2} T^{3}}{6}-\frac{\beta a T^{3}}{3}-\frac{\beta b T^{4}}{8}+\frac{a b T^{4}}{8}+\frac{a^{2} T^{3}}{6}+\frac{b^{2} T^{5}}{40}+\cdots\right]+I_{0}
\end{aligned}
$$

So that

$$
\begin{aligned}
I_{0}= & K\left[T+h T-\frac{\beta T^{2}}{2}+\frac{a T^{2}}{2}+\frac{b T^{3}}{6}+\frac{h^{2} T}{2}-\frac{h \beta T^{2}}{2}+\frac{h a T^{2}}{2}+\frac{b h T^{3}}{6}\right. \\
& \left.+\frac{\beta^{2} T^{3}}{6}-\frac{\beta a T^{3}}{3}-\frac{\beta b T^{4}}{8}+\frac{a b T^{4}}{8}+\frac{a^{2} T^{3}}{6}+\frac{b^{2} T^{5}}{40}+\cdots\right]
\end{aligned}
$$

Substituting Equation (8) into Equation (6) gives 


$$
\begin{aligned}
I(t) \mathrm{e}^{a t+\frac{1}{2} b t^{2}}= & -K\left[t+h t-\frac{\beta t^{2}}{2}+\frac{a t^{2}}{2}+\frac{b t^{3}}{6}+\frac{h^{2} t}{2}-\frac{h \beta t^{2}}{2}+\frac{h a t^{2}}{2}\right. \\
& \left.+\frac{b h t^{3}}{6}+\frac{\beta^{2} t^{3}}{6}-\frac{\beta a T^{3}}{3}-\frac{\beta b t^{4}}{8}+\frac{a^{2} t^{3}}{6}+\frac{b^{2} t^{5}}{40}+\cdots\right] \\
& +K\left[T+h T-\frac{\beta T^{2}}{2}+\frac{a T^{2}}{2}+\frac{b T^{3}}{6}+\frac{h^{2} T}{2}-\frac{h \beta T^{2}}{2}+\frac{h a T^{2}}{2}\right. \\
& \left.+\frac{b h T^{3}}{6}+\frac{\beta^{2} T^{3}}{6}-\frac{\beta a T^{3}}{3}-\frac{\beta b T^{4}}{8}+\frac{a b T^{4}}{8}+\frac{a^{2} T^{3}}{6}+\frac{b^{2} T^{5}}{40}+\cdots\right] \\
\Rightarrow I(t)= & K \mathrm{e}-a t-\frac{1}{2} b t^{2}\left[(T-t)+h(T-t)-\frac{\beta}{2}\left(T^{2}-t^{2}\right)+\frac{a}{2}\left(T^{2}-t^{2}\right)\right. \\
+ & \frac{b}{6}\left(T^{3}-t^{3}\right)+\frac{h^{2}}{2}(T-t)-\frac{h \beta}{2}\left(T^{2}-t^{2}\right)+\frac{h a}{2}\left(T^{2}-t^{2}\right) \\
+ & \frac{h b}{6}\left(T^{3}-t^{3}\right)+\frac{\beta^{2}}{6}\left(T^{3}-t^{3}\right)-\frac{a \beta}{3}\left(T^{3}-t^{3}\right)-\frac{b \beta}{8}\left(T^{4}-t^{4}\right) \\
+ & \left.\frac{a b}{8}\left(T^{4}-t^{4}\right)+\frac{a^{2}}{6}\left(T^{3}-t^{3}\right)+\frac{b^{2}}{40}\left(T^{5}-t^{5}\right)+\cdots\right]
\end{aligned}
$$

The total demand during the cycle period $[0, T]$ is given as follows:

$$
\int_{0}^{T} D(t) \mathrm{d} t=\int_{0}^{T} K \mathrm{e}^{h-\beta t} \mathrm{~d} t=\frac{K}{-\beta}\left[\mathrm{e}^{h-\beta t}\right]_{0}^{T}=\frac{K \mathrm{e}^{h}}{-\beta}\left[\mathrm{e}^{-\beta T}-1\right]
$$

The number of deteriorated units is given as initial order quantity minus the total demand in the cycleperiod $[0, T]$. Thus the number of deteriorated units is

$$
\begin{gathered}
I_{0}-\int_{0}^{T} D(t) \mathrm{d} t \\
=K\left[T+h T-\frac{\beta T^{2}}{2}+\frac{a T^{2}}{2}+\frac{b T^{3}}{6}+\frac{h^{2} T}{2}-\frac{h \beta T^{2}}{2}+\frac{h a T^{2}}{2}+\frac{b h T^{3}}{6}+\frac{\beta^{2} T^{3}}{6}\right. \\
\left.-\frac{\beta a T^{3}}{3}-\frac{\beta b T^{4}}{8}+\frac{a b T^{4}}{8}+\frac{a^{2} T^{3}}{6}+\frac{b^{2} T^{5}}{40}+\cdots\right]-\left\{\frac{K \mathrm{e}^{h}}{-\beta}\left[\mathrm{e}^{-\beta T}-1\right]\right\} \\
=K\left[T+h T-\frac{\beta T^{2}}{2}+\frac{a T^{2}}{2}+\frac{b T^{3}}{6}+\frac{h^{2} T}{2}-\frac{h \beta T^{2}}{2}+\frac{h a T^{2}}{2}+\frac{b h T^{3}}{6}+\frac{\beta^{2} T^{3}}{6}\right. \\
\left.-\frac{\beta a T^{3}}{3}-\frac{\beta b T^{4}}{8}+\frac{a b T^{4}}{8}+\frac{a^{2} T^{3}}{6}+\frac{b^{2} T^{5}}{40}+\frac{\mathrm{e}^{h-\beta T}}{\beta}-\frac{\mathrm{e}^{h}}{\beta}+\cdots\right]
\end{gathered}
$$

Deterioration cost $(\mathrm{DC})$ for the cycle $[O, T]=A_{c} \times$ (the number of deteriorated units)

$$
\begin{aligned}
= & A_{c} K\left[T+h T-\frac{\beta T^{2}}{2}+\frac{a T^{2}}{2}+\frac{b T^{3}}{6}+\frac{h^{2} T}{2}-\frac{h \beta T^{2}}{2}+\frac{h a T^{2}}{2}+\frac{b h T^{3}}{6}+\frac{\beta^{2} T^{3}}{6}\right. \\
& \left.-\frac{\beta a T^{3}}{3}-\frac{\beta b T^{4}}{8}+\frac{a b T^{4}}{8}+\frac{a^{2} T^{3}}{6}+\frac{b^{2} T^{5}}{40}+\frac{\mathrm{e}^{h-\beta T}}{\beta}-\frac{\mathrm{e}^{h}}{\beta}+\cdots\right]
\end{aligned}
$$

To find the total inventory holding cost (IHC) for the cycle $[O, T]$ we note that 


$$
\begin{aligned}
& \mathrm{e}^{-a t-\frac{1}{2} b t^{2}}=1+\frac{-a t-\frac{1}{2} b t^{2}}{1 !}+\frac{\left(-a t-\frac{1}{2} b t^{2}\right)^{2}}{2 !}+\cdots \\
& =1-a t-\frac{1}{2} b t^{2}+\frac{a^{2} t^{2}+a b t^{3}+\frac{1}{4} b^{2} t^{4}}{2}+\cdots \\
& =1-a t-\frac{1}{2} b t^{2}+\frac{a^{2} t^{2}}{2}+\frac{a b t^{3}}{2}+\frac{b^{2} t^{4}}{8}+\cdots \\
& \therefore I(t)=K\left[( 1 - a t - \frac { 1 } { 2 } b t ^ { 2 } + \frac { a ^ { 2 } t ^ { 2 } } { 2 } + \frac { a b t ^ { 3 } } { 2 } + \frac { b ^ { 2 } t ^ { 4 } } { 8 } + \cdots ) \left\{\left(1+h+\frac{h^{2}}{2}\right)(T-t)\right.\right. \\
& +\left(-\frac{\beta}{2}+\frac{a}{2}-\frac{h \beta}{2}+\frac{h a}{2}\right)\left(T^{2}-t^{2}\right)+\left(\frac{b}{6}+\frac{h b}{6}+\frac{\beta^{2}}{6}-\frac{\beta a}{3}+\frac{a^{2}}{6}\right)\left(T^{3}-t^{3}\right) \\
& \left.\left.+\left(\frac{-\beta b}{8}+\frac{a b}{8}\right)\left(T^{4}-t^{4}\right)+\frac{b^{2}}{40}\left(T^{5}-t^{5}\right)+\cdots\right\}\right] \\
& =K\left[\frac{1}{2}\left(2+2 h+h^{2}\right)(T-t)+\frac{1}{2}(-\beta+a-h \beta+h a)\left(T^{2}-t^{2}\right)\right. \\
& +\frac{1}{6}\left(b+h b+\beta^{2}-2 a \beta+a^{2}\right)\left(T^{3}-t^{3}\right)+\frac{b}{8}(-\beta+a)\left(T^{4}-t^{4}\right) \\
& +\frac{b^{2}}{40}\left(T^{5}-t^{5}\right)-\frac{a}{2}\left(2+2 h+h^{2}\right)\left(T t-t^{2}\right)-\frac{a}{2}(-\beta+a-h \beta+h a)\left(T^{2} t-t^{3}\right) \\
& -\frac{a}{6}\left(b+h b+\beta^{2}-2 a \beta+a^{2}\right)\left(T^{3} t-t^{4}\right)-\frac{a b}{8}(-\beta+a)\left(T^{4} t-t^{5}\right) \\
& -\frac{a b^{2}}{40}\left(T^{5} t-t^{6}\right)+\frac{1}{4}\left(a^{2}-b\right)\left(2+2 h+h^{2}\right)\left(T t^{2}-t^{3}\right) \\
& +\frac{1}{4}\left(a^{2}-b\right)(-\beta+a-h \beta+h a)\left(T^{2} t^{2}-t^{4}\right) \\
& +\frac{1}{12}\left(a^{2}-b\right)\left(b+h b+\beta^{2}-2 a \beta+a^{2}\right)\left(T^{3} t^{2}-t^{5}\right) \\
& +\frac{b}{16}\left(a^{2}-b\right)(-\beta+a)\left(T^{4} t^{2}-t^{6}\right)-\frac{b^{3}}{80}\left(T^{5} t^{2}-t^{7}\right)+\frac{a^{2} b^{2}}{80}\left(T^{5} t^{2}-t^{7}\right) \\
& +\frac{a b}{4}\left(2+2 h+h^{2}\right)\left(T t^{3}-t^{4}\right)+\frac{a b}{4}(-\beta+a-h b+h a)\left(T^{2} t^{3}-t^{5}\right) \\
& +\frac{a b}{12}\left(b+h b+\beta^{2}-2 a \beta+a^{2}\right)\left(T^{3} t^{3}-t^{6}\right)+\frac{a b^{2}}{16}(-\beta+a)\left(T^{4} t^{3}-t^{7}\right) \\
& +\frac{a b^{3}}{80}\left(T^{5} t^{3}-t^{8}\right)+\frac{b^{2}}{16}\left(2+2 h+h^{2}\right)\left(T t^{4}-t^{5}\right) \\
& +\frac{b^{2}}{16}(-\beta+a-h \beta+h a)\left(T^{2} t^{4}-t^{6}\right) \\
& +\frac{b^{2}}{48}\left(b+h b+\beta^{2}-2 a \beta+a^{2}\right)\left(T^{3} t^{4}-t^{7}\right) \\
& \left.+\frac{b^{3}}{64}(-\beta+a)\left(T^{4} t^{4}-t^{8}\right)+\frac{b^{4}}{320}\left(T^{5} t^{4}-t^{9}\right)+\cdots\right]
\end{aligned}
$$

Therefore the total inventory holding cost $(I H C)$ is given by 


$$
I H C=i C \int_{0}^{T} I(t) \mathrm{d} t
$$

Substituting Equation (15) into Equation (16) gives

$$
\begin{aligned}
& I H C=i C K \int_{0}^{T}\left[\frac{1}{2}\left(2+2 h+h^{2}\right)(T-t)+\frac{1}{2}(-\beta+a-h \beta+h a)\left(T^{2}-t^{2}\right)\right. \\
& +\frac{1}{6}\left(b+h b+\beta^{2}-2 a \beta+a^{2}\right)\left(T^{3}-t^{3}\right)+\frac{b}{8}(-\beta+a)\left(T^{4}-t^{4}\right) \\
& +\frac{b^{2}}{40}\left(T^{5}-t^{5}\right)-\frac{a}{2}\left(2+2 h+h^{2}\right)\left(T t-t^{2}\right) \\
& -\frac{a}{2}(-\beta+a-h \beta+h a)\left(T^{2} t-t^{3}\right) \\
& -\frac{a}{6}\left(b+h b+\beta^{2}-2 a \beta+a^{2}\right)\left(T^{3} t-t^{4}\right)-\frac{a b}{8}(-\beta+a)\left(T^{4} t-t^{5}\right) \\
& -\frac{a b^{2}}{40}\left(T^{5} t-t^{6}\right)+\frac{1}{4}\left(a^{2}-b\right)\left(2+2 h+h^{2}\right)\left(T t^{2}-t^{3}\right) \\
& +\frac{1}{4}\left(a^{2}-b\right)(-\beta+a-h \beta+h a)\left(T^{2} t^{2}-t^{4}\right) \\
& +\frac{1}{12}\left(a^{2}-b\right)\left(b+h b+\beta^{2}-2 a \beta+a^{2}\right)\left(T^{3} t^{2}-t^{5}\right) \\
& +\frac{b}{16}\left(a^{2}-b\right)(-\beta+a)\left(T^{4} t^{2}-t^{6}\right)-\frac{b^{3}}{80}\left(T^{5} t^{2}-t^{7}\right)+\frac{a^{2} b^{2}}{80}\left(T^{5} t^{2}-t^{7}\right) \\
& +\frac{a b}{4}\left(2+2 h+h^{2}\right)\left(T t^{3}-t^{4}\right)+\frac{a b}{4}(-\beta+a-h b+h a)\left(T^{2} t^{3}-t^{5}\right) \\
& +\frac{a b}{12}\left(b+h b+\beta^{2}-2 a \beta+a^{2}\right)\left(T^{3} t^{3}-t^{6}\right)+\frac{a b^{2}}{16}(-\beta+a)\left(T^{4} t^{3}-t^{7}\right) \\
& +\frac{a b^{3}}{80}\left(T^{5} t^{3}-t^{8}\right)+\frac{b^{2}}{16}\left(2+2 h+h^{2}\right)\left(T t^{4}-t^{5}\right) \\
& +\frac{b^{2}}{16}(-\beta+a-h \beta+h a)\left(T^{2} t^{4}-t^{6}\right) \\
& +\frac{b^{2}}{48}\left(b+h b+\beta^{2}-2 a \beta+a^{2}\right)\left(T^{3} t^{4}-t^{7}\right) \\
& \left.+\frac{b^{3}}{64}(-\beta+a)\left(T^{4} t^{4}-t^{8}\right)+\frac{b^{4}}{320}\left(T^{5} t^{4}-t^{9}\right)+\cdots\right] \mathrm{d} t \\
& \therefore I H C=i C K\left[\frac{T^{2}}{4}\left(2+2 h+h^{2}\right)+\frac{T^{3}}{12}\left(-4 \beta+4 a-4 h \beta+4 h a-2 a-2 a h-a h^{2}\right)\right. \\
& +\frac{T^{4}}{48}\left(6 b+6 h b+6 \beta^{2}-12 \beta a+6 a^{2}+6 a \beta-6 a^{2}+6 a h \beta-6 a^{2} h+2 a^{2}\right. \\
& \left.+2 h a^{2}+a^{2} h^{2}-2 b-2 b h-b h^{2}\right)+\frac{T^{5}}{240}(-24 b \beta+24 a b-12 a b-12 h a b \\
& -12 a \beta^{2}+24 a^{2} \beta-12 a^{3}-8 a^{2} \beta+8 a^{3}-8 a^{2} h \beta+8 a^{3} h+8 b \beta-8 a b \\
& \left.+8 b h \beta-8 a b h+6 a b+6 a b h+3 a b h^{2}\right)+\frac{T^{6}}{1440}\left(30 b^{2}+60 a b \beta-60 a^{2} b\right.
\end{aligned}
$$




$$
\begin{aligned}
& +20 a^{2} b+20 a^{2} h b+20 a^{2} \beta^{2}-40 a^{3} \beta+20 a^{4}-20 b^{2}-20 b^{2} h-20 b \beta^{2} \\
& +40 a b \beta-20 a^{2} b-30 a b \beta+30 a^{2} b-30 a b h \beta+30 a^{2} b h+6 b^{2}+6 b^{2} h \\
& \left.+3 b^{2} h^{2}\right)+\frac{T^{7}}{1680}\left(-15 a b^{2}-20 a^{2} b \beta+20 a^{3} b+20 b^{2} \beta-20 a b^{2}+15 a b^{2}\right. \\
& \left.+15 a b^{2} h+15 a b \beta^{2}-30 a^{2} b \beta+15 a^{3} b-6 b^{2} \beta+6 a b^{2}-6 b^{2} h \beta+6 a b^{2} h\right) \\
& +\frac{T^{8}}{1920}\left(-5 b^{3}+5 a^{2} b^{2}-15 a b^{2} \beta+15 a^{2} b^{2}+3 b^{3}+3 b^{3} h+3 b^{2} \beta^{2}\right. \\
& \left.\left.-6 a b^{2} \beta+3 a^{2} b^{2}\right)+\frac{T^{9}}{2880}\left(5 a b^{3}-4 b^{3} \beta+4 a b^{3}\right)+\frac{T^{10} b^{4}}{3200}+\cdots\right]
\end{aligned}
$$

Letting $P_{2}=\frac{1}{4}\left(2+2 h+h^{2}\right), \quad P_{3}=\frac{1}{12}\left(-4 \beta+2 a-4 h \beta+2 a h-a h^{2}\right)$,

$$
\begin{gathered}
P_{4}=\frac{1}{48}\left(4 b+4 h b+6 \beta^{2}-6 \beta a+2 a^{2}+6 a h \beta-4 a^{2} h+a^{2} h^{2}-b h^{2}\right), \\
P_{5}=\frac{1}{240}\left(-16 b \beta+10 a b-14 h a b-12 a \beta^{2}+16 a^{2} \beta-4 a^{3}\right. \\
\left.-8 a^{2} h \beta+8 h a^{3}+8 h b \beta+3 a b h^{2}\right), \\
P_{6}=\frac{1}{1440}\left(16 b^{2}+70 a b \beta-30 a^{2} b+50 a^{2} h b+20 a^{2} \beta^{2}-40 a^{3} \beta\right. \\
\left.+20 a^{4}-14 h b^{2}-20 b \beta^{2}-30 a b h \beta+3 b^{2} h^{2}\right), \\
P_{7}=\frac{1}{1680}\left(-14 a b^{2}-50 a^{2} b \beta+35 a^{3} b+14 b^{2} \beta+21 a b^{2} h+15 a b \beta^{2}-6 \beta b^{2} h\right), \\
P_{8}=\frac{1}{1920}\left(-2 b^{3}+23 a^{2} b^{2}-21 a b^{2} \beta+3 b^{3} h+3 b^{2} \beta^{2}\right), \\
P_{9}=\frac{1}{2880}\left(9 a b^{3}-4 b^{3} \beta\right) \text { and } P_{10}=\frac{b^{4}}{3200} \text { gives }=i C K\left[P_{2} T^{2}+P_{3} T^{3}+P_{4} T^{4}+P_{5} T^{5}+P_{6} T^{6}+P_{7} T^{7}+P_{8} T^{8}+P_{9} T^{9}+P_{10} T^{10}\right],
\end{gathered}
$$

However, the total variable cost $=$ Ordering cost $(O C)+$ Deterioration cost + Inventory Holding cost $(I H C)$ and the total variable Cost per unit time $T C(T)$ is $=\frac{\text { Total variable cost }}{\text { Time }}$

$$
\begin{aligned}
\therefore T C(T)= & \frac{N_{0}}{T}+\frac{A_{c} K}{T}\left[T+h T-\frac{\beta T^{2}}{2}+\frac{a T^{2}}{2}+\frac{b T^{3}}{6}+\frac{h^{2} T}{2}-\frac{h \beta T^{2}}{2}+\frac{h a T^{2}}{2}\right. \\
& +\frac{b h T^{3}}{6}+\frac{\beta^{2} T^{3}}{6}-\frac{\beta a T^{3}}{3}-\frac{\beta b T^{4}}{8}+\frac{a b T^{4}}{8}+\frac{a^{2} T^{3}}{6}+\frac{b^{2} T^{5}}{40}-\frac{\mathrm{e}^{h-\beta T}}{\beta} \\
& \left.-\frac{\mathrm{e}^{h}}{\beta}+\cdots\right]+\frac{i C K}{T}\left[P_{2} T^{2}+P_{3} T^{3}+P_{4} T^{4}+P_{5} T^{5}+P_{6} T^{6}+P_{7} T^{7}\right. \\
& \left.\left.+P_{8} T^{8}+P_{9} T^{9}+P_{10} T^{10}\right]\right]
\end{aligned}
$$

The main objective is to find the cycle length $T$ that will give the minimum variable cost per unit time. The necessary and sufficient conditions to minimize 
$T C(T)$ are respectively,

$$
\frac{\mathrm{d} T C(T)}{\mathrm{d} T}=0 \text { and } \frac{\mathrm{d}^{2} T C(T)}{\mathrm{d} T^{2}}>0
$$

Therefore to satisfy the necessary condition we have to differentiate Equation (19) with respect to $T$, as follows

$$
\begin{aligned}
\frac{\mathrm{d} T C(T)}{\mathrm{d} T}= & -\frac{N_{0}}{T^{2}}+A_{c} K\left[-\frac{\beta}{2}+\frac{a}{2}+\frac{b T}{3}-\frac{h \beta}{2}+\frac{h a}{2}+\frac{h b T}{3}+\frac{\beta^{2} T}{3}-\frac{2 \beta a T}{3}\right. \\
& \left.-\frac{3 \beta b T^{2}}{8}+\frac{3 a b T^{2}}{8}+\frac{a^{2} T}{3}+\frac{b^{2} T^{3}}{10}+\frac{\mathrm{e}^{h-\beta T}}{T}+\frac{\mathrm{e}^{h-\beta T}}{\beta T^{2}}+\frac{\mathrm{e}^{h}}{\beta T^{2}}+\cdots\right] \\
& +i C K\left[P_{2}+2 P_{3} T+3 P_{4} T^{2}+4 P_{5} T^{3}+5 P_{6} T^{4}+6 P_{7} T^{5}+7 P_{8} T^{6}\right. \\
& \left.+8 P_{9} T^{7}+9 P_{10} T^{8}\right]
\end{aligned}
$$

We now equate Equation (20) to zero and simplify by multiplying with $\left[-120 T^{2} \beta\right]$ on both sides in order to determine the $T$ which minimizes the variable cost per unit time as follows:

$$
\begin{aligned}
& 120 \beta N_{0}+60 A_{c} K \beta^{2} T^{2}-60 A_{c} K a \beta T^{2}-40 A_{c} K b \beta T^{3}+60 A_{c} K h \beta^{2} T^{2} \\
& -60 A_{c} K h a \beta T^{2}-40 A_{c} K b h \beta T^{3}-40 A_{c} K \beta^{3} T^{3}+80 A_{c} K a \beta^{2} T^{3} \\
& +45 A_{c} K b \beta^{2} T^{4}-45 A_{c} K a b \beta T^{4}-40 A_{c} K a^{2} \beta T^{3}-12 A_{c} K b^{2} \beta T^{5} \\
& -120 A_{c} K \beta T \mathrm{e}^{h-\beta T}-120 A_{c} K \mathrm{e}^{h-\beta T}-120 A_{c} K \mathrm{e}^{h}-120 i C K P_{2} \beta T^{2} \\
& -240 i C K P_{3} \beta T^{3}-360 i C K P_{4} \beta T^{4}-480 i C K P_{5} \beta T^{5}-600 i C K P_{6} \beta T^{6} \\
& -720 i C K P_{7} \beta T^{7}-840 i C K P_{8} \beta T^{8}-960 i C K P_{9} \beta T^{9}-1080 i C K P_{10} \beta T^{10}+\cdots=0
\end{aligned}
$$

The value of $T$ obtained, gives the minimum cost provided it satisfies the following condition

$$
\frac{\mathrm{d}^{2} T C(T)}{\mathrm{d} T^{2}}>0 .
$$

Equation (21) is highly nonlinear and therefore difficult to solve by any analytic method. Likewise the same problem will exist in trying to check the inequality in (22) above. However, in all our examples below, we use direct search method with the help of EXCEL to obtain the root of the equation and also confirm that the sufficient Condition (21) is satisfied.

\section{Numerical Example}

\section{Example}

To illustrate the model developed an example is considered based on the following values of parameters: $N_{0}=\$ 5000$ per order, $K=250, C=\$ 2.50$ per unit, $\beta=1, a=0.8, b=2, i=0.1$ per Naira per unit time, $A_{c}=\$ 3.00$, and $h=0.5$, taking some parameter values from Dash et al. (2014) [17]. Substituting and simplifying the above parameters into Equation (21), gives $T^{*}=1.34247 \quad(490$ days). On substitution of this optimal value $T^{*}$ in Equations (19) and (8), we obtain the minimum total cost per unit time $T C^{*}=\$ 4578.88$ and economic order quantity $I_{0}^{*}=852.6601216$ units. Note that the $T^{*}$ value satisfies 


$$
\frac{\mathrm{d}^{2} T C(T)}{\mathrm{d} T^{2}}>0
$$

\section{Sensitivity Analysis}

A sensitivity analysis was carried out to study the effect of changes in the values of the system parameters $N_{0}, K, \beta, a, b, C, i, A_{c}$ and $h$ on the optimal length of the cycle $\left(T^{*}\right)$, the economic order quantity $\left(I_{0}^{*}\right)$ and the minimum total cost per unit time $\left(T C^{*}\right)$. The sensitivity analysis was performed for each of the parameters by changing its value by $50 \%, 25 \%, 5 \%, 2 \%,-2 \%,-5 \% .-25 \%,-50 \%$, while keeping the remaining parameters at their original values. The analysis showed the following:

1) With increase in the value of the parameter $a$, the values of $T^{*}$ decrease while $T C^{*}$ and $I_{0}^{*}$ increase. This is because when a increases, deterioration increases and so to avoid much deterioration the model forces $T^{*}$ to decrease. $I_{0}^{*}$ increases probably to compensate for the deteriorated units. $T C^{*}$ increases due to the cost of deterioration. The increase/decrease in the values is moderate hence the decision variables are moderately sensitive to changes in $a$.

2) With increase in the value of parameter $b$, the values of $T^{*}$ and $I_{0}^{*}$ decrease while the value of $T C^{*}$ increases. This is expected because when $b$ increases, deterioration with respect to time also increases and so the model forces $T^{*}$ and $I_{0}^{*}$ to decrease. $T C^{*}$ increases due to the cost of deterioration. The decreases/increase in the values are moderate hence the decision variables are moderately sensitive to changes in $b$.

3) With increase in the value of parameter $A_{\odot}$ the values of $T^{*}$ and $I_{0}^{*}$ decrease while $T C^{*}$ increases. This is also expected since when the cost of a deteriorated unit increases then the model will avoid much quantity in a supply and so both $T^{*}$ and $I_{0}^{*}$ decrease. $T C^{*}$ increases because of the cost of deteriorated items. The increase/decrease in the values is moderate hence the decision variables are moderately sensitive to changes in $A_{c}$

4) With increase in the value of the parameter $\beta$, the values of $T^{*}$ and $I_{0}^{*}$ increase while $T C^{*}$ is not stable. This is not expected because when $\beta$ increases, it is expected that $T^{*}$ and $I_{0}^{*}$ should decrease. The fact that $T C^{*}$ is not stable indicates that the model tries to adjust $T C^{*}$ to the minimum value, at the expense of increasing $T^{*}$ and $I_{0}^{*}$. The increases/decrease in the values are low hence the variables are lowly sensitive to changes in $\beta$.

5) With increase in the value of the parameter $N_{0}$, the values of $T^{*}, T C^{*}$ and $I_{0}^{*}$ increase. This is also expected since when ordering cost increases then the model will avoid more orders and so both $T^{*}$ and $I_{0}^{*}$ increase. $T C^{*}$ will however increase due to increase in stockholding cost. The increases in the values are high hence the decision variables $T^{*}, T C^{*}$ and $I_{0}^{*}$ are highly sensitive to changes in $N_{0}$.

6) With increase in the value of parameter $K$, the values of $T C^{*}$ and $I_{0}^{*}$ increase while $T^{*}$ decreases. This is because when $K$ increases, there will be more 
demand and so the economic order quantity $\left(I_{0}^{*}\right)$ will increase. This will result in increase in the optimal total cost $\left(T C^{*}\right)$. The cycle period $\left(T^{*}\right)$ decreases probably as a result of the model trying to avoid much deterioration. The increases/decrease in the values are moderate hence the decision variables are moderately sensitive to changes in $K$.

7) With increase in the value of parameter $h$, the values of $T C^{*}$ and $I_{0}^{*}$ increase while $T^{*}$ decreases. This is because as the demand increases the economic order quantity also increases, hence the total variable cost, $T C^{*}$ also increases. On the other hand however, the cycle period decreases which is probably due to higher demand. The increase in the values is moderate hence the decision variables are moderately sensitive to changes in $h$.

8) With increase in the value of parameter $i$, the values of $T^{*}$ and $I_{0}^{*}$ decrease while $T C^{*}$ increases. This is expected because when the inventory carrying charge, $i$ is increased there will be more stockholding cost so the model will avoid that by reducing quantity in an order which results in decreasing $I_{0}^{*}$ and $T^{*} . T C^{*}$ increases due to the increase in the carrying charge (i). The increase/decrease in the values is moderate hence the decision variables are moderately sensitive to changes in $i$.

With increase in the value of parameter $C$, the values of $T^{*}$ and $I_{0}^{*}$ decrease while $T C^{*}$ increases. This is also expected because when the unit cost of an item, $C$, is increased there will be more stockholding cost so the model will avoid that by reducing quantity in an order, which results in decreasing $I_{0}^{*}$ and $T^{*}$. $T C^{*}$ increases due to the increase in the unit cost. The increase/decrease in the values is moderate hence the decision variables are moderately sensitive to changes in $C$.

\section{References}

[1] Wee, H.M. (1993) Economic Order Quantity Model for Deteriorating Items with partial Back Ordering. Computer and Industrial Engineering, 24, 449-458.

[2] Ghare, P.N and Schrader, G.P. (1963) A Model for Exponential Decaying Inventory. Journal of Industrial Engineering, 14, 238-243.

[3] Covert, R.B. and Philip, G.S. (1973) An EOQ Model with Weibull Distribution Deterioration. AIIE Transactions, 5, 323-326. https://doi.org/10.1080/05695557308974918

[4] Shah, Y.K. and Jaiswal, M.C. (1977) An Order-Level Inventory Model for a System with Constant Rate of Deterioration. Operational Research, 14, 174-184.

[5] Aggarwal, S.P. (1978) A Note on an Order-Level Model for a System with Constant Rate of Deterioration. Operational Research, 15, 184-187.

[6] Dave, U. and Patel, L.K. (1981) ( $T, S_{i}$ ) Policy inventory Model for Deteriorating items with Time-Proportional Demand. Journal of the Operational Research Society, 32, 137-142. https://doi.org/10.1057/jors.1981.27

[7] Hollier, R.H. and Mark, K.L. (1983) Inventory Replenishment Policies for Deteriorating Items in a Declining Market. International Journal of Production Research, 21, 813-836.

[8] Hariga, M. and Benkherouf, L. (1994) Operational and Heuristic Replenishment 
Models for Deteriorating Items with Exponential Time Varying Demand. European Journal of Operational Research, 79, 123-137. https://doi.org/10.1016/0377-2217(94)90400-6

[9] Wee, H.M. (1995) A Deterministic Lot Size Inventory Model for Deteriorating Items with Shortages and Declining Market. Computers and operations Research, 22, 345-356.

[10] Wee, H.M. (1995) Joint Pricing and Replenishment policy for Deteriorating Inventory with Declining Market. International Journal of Production Economic, 40, 163-171.

[11] Goyal, S.K. and Giri, B.C. (2001) Recent Trends in Modelling of Deteriorating Inventory. European Journal of Operational Research, 134, 1-16.

https://doi.org/10.1016/S0377-2217(00)00248-4

[12] Ouyang L. Y., Wu K. S. and Chenga M. C. (2005), An Inventory Model for Deteriorating Items with Exponential Declining Demand and partial Backlogging. Yugoslav Journal of Operations Research, 15, 277-288. https://doi.org/10.2298/YJOR0502277O

[13] Shah, N.H. and Pandy, P. (2008) Optimal Ordering Policy for the Time Dependent Value When Delay in Payments Is Permissible. Revista Investigation Operational, 2, 117-129.

[14] He, Y. and He, J. (2010) A Production Model for Deteriorating Inventory Items with Production Disruptions, Hindawi Publishing Corporation Discrete Dynamics in Nature and Society, 2010(189017), 14.

[15] Kumar, S., Kumar, P. and Saini, M. (2012) An Order Level Inventory Model for Deteriorating Items with Quadratic Demand Rate and Variable Holding Cost. International Journal of Scientific Research Engineering and Technology (IJSRET), 1, 253-263.

[16] Singh, T. and Pattnayak, H. (2013) An EOQ Model for a Deteriorating Item with Time Dependent Quadratic Demand and Variable Deterioration under Permissible Delay in Payment. Applied Mathematical Science, 7, 2939-2951.

https://doi.org/10.12988/ams.2013.13261

[17] Dash, B.P., Sing, T. and Pattanayak, H. (2014), An Inventory Model for Deteriorating Items with Exponential Declining Demand and Time-Varying Holding Cost. American Journal of Operational Research, 4, 1-7. https://doi.org/10.4236/ajor.2014.41001

[18] Aliyu, I. and Sani, B. (2018) An Inventory Model for Deteriorating Items with Generalised Exponential Decreasing Demand, Constant Holding Cost and Timevarying Deterioration Rate. American Journal of Operations Research, 8, 1-16.

[19] Wataru (2014) Power Series and Estimation of Integrals-Calculus $\backslash$ Socratic. https://socratic.org/calculus/power-series-and-estimation (Retrieved on 10/02/2020). 